\title{
Fistule cutanée d'origine dentaire : recherche de la dent causale
}

\author{
Lamia Oualha ${ }^{1, \star}$, Sana Bagga ${ }^{1}$, Nabiba Douki ${ }^{1}$, Faten Ben Amor ${ }^{2}$, Jamil Selmi ${ }^{2}$ \\ 1 Service de Médecine dentaire, EPS Sahloul, Sousse, Tunisie \\ 2 Médecine et Chirurgie buccales, Clinique universitaire dentaire, Monastir, Tunisie
}

(Reçu le $1^{\text {er }}$ octobre 2010, accepté le 17 novembre 2010)

Mots clés :

fistule /

cutané /

odontogène

\begin{abstract}
Résumé - Les fistules cutanées d'origine dentaire peuvent constituer un défi diagnostique. En l'absence de signes endobuccaux, l'origine dentaire n'est pas toujours évoquée d'emblée. Dans quelques cas, les examens clinique et radiologique ne permettent pas de déterminer l'étiologie et la dent causale. Faute de diagnostic correct, on est amené à réaliser un traitement inapproprié et inefficace.

Les auteurs présentent un cas de fistule cutanée génienne basse, secondaire à une nécrose pulpaire de la première molaire et/ou à une péricoronarite sur la dent de sagesse. Cette double étiologie probable a prolongé le traitement et retardé la guérison.

Lorsque la fistule cutanée d'origine dentaire n'est pas accompagnée de symptômes dentaires évidents et qu'il existe plusieurs dents causales éventuelles, il est parfois difficile d'identifier la dent responsable. La persistance de la fistule après le traitement initial a conduit à rechercher une autre dent causale.
\end{abstract}

\begin{abstract}
Challenging diagnosis face to cutaneous dental sinus tract. Cutaneous draining sinus tracts of dental origin often are a diagnostic challenge. A delay in correctly diagnosing these types of lesions can result in ineffective and inappropriate treatment.

The authors present a case of cutaneous sinus tract on the lower cheek secondary to pulpal necrosis of the first molar and/or pericoronitis or the third molar. This double etiology has extended the treatment and delayed the healing.

As patients with cutaneous facial sinus tracts of dental origin often do not have obvious dental symptoms, possible double dental etiology may be overlooked. Early correct diagnosis and treatment of these lesions can help prevent unnecessary surgical treatment.
\end{abstract}

La fistule cutanée d'origine dentaire représente une voie de drainage plus ou moins longue pour une infection dentaire [1]; il s'agit d'une entité connue et décrite depuis fort longtemps [2]. Cependant, errance diagnostique et retard de prise en charge thérapeutique sont encore fréquents, même en Médecine bucco-dentaire [3,4]. Parfois, elle est attribuée à une autre cause et tout traitement local, le plus souvent l'exérèse de la lésion cutanée, est rapidement suivi d'une récidive [5].

A travers ce travail, l'accent est mis sur la nécessité d'établir un diagnostic étiologique correct afin d'adopter une attitude thérapeutique adéquate qui, seule, permet d'obtenir la guérison et d'éviter au patient un préjudice esthétique.

\footnotetext{
^Correspondance : lamia.oualha@gmail.com
}

\section{Cas clinique}

Un patient âgé de 20 ans, en bon état général, consulte pour une lésion jugale inesthétique. L'examen exobuccal révèle une fistule cutanée productive, non rétractile, évoluant depuis un mois, associée à une inflammation subaiguë (Fig. 1). Il n'y a pas de tuméfaction génienne ni submandibulaire. La palpation indolore fait sourdre des sérosités. L'ouverture buccale est normale. L'examen endobuccal montre des caries sur 36 et 37 ; la 38 est enclavée, sans signes d'inflammation ni de suppuration (Fig. 2). Le test de vitalité est négatif sur la 36 , positif sur la 37 . La percussion axiale n'est pas douloureuse. Le vestibule inférieur gauche est libre et la muqueuse d'aspect normal. La recherche d'un cordon fistuleux par mise en tension de la joue est négative.

La radiographie rétro-alvéolaire montre un léger élargissement desmodontal dans la région de la furcation de la 36 et 


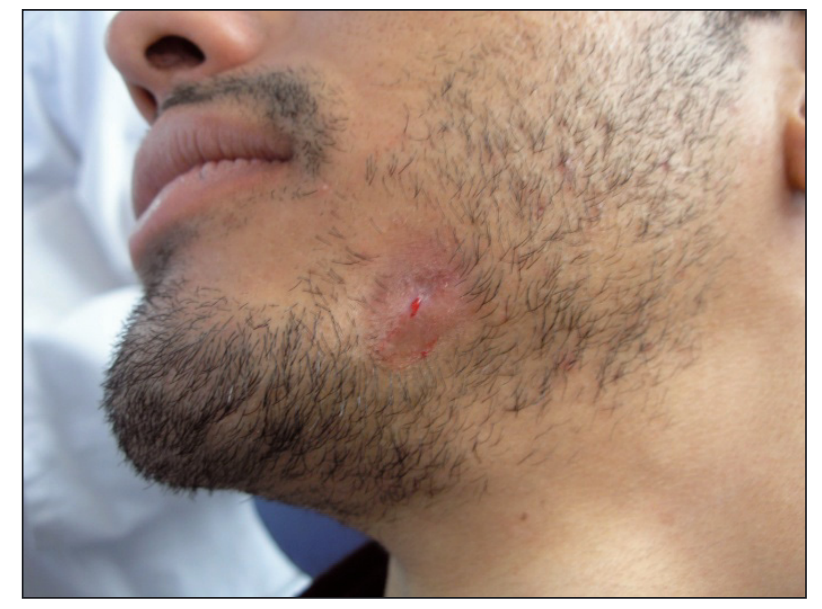

Fig. 1. Fistule cutanée génienne basse gauche au centre d'une inflammation subaiguë.

Fig. 1. Cutaneous sinus tract left bottom center of a subacute inflammation.

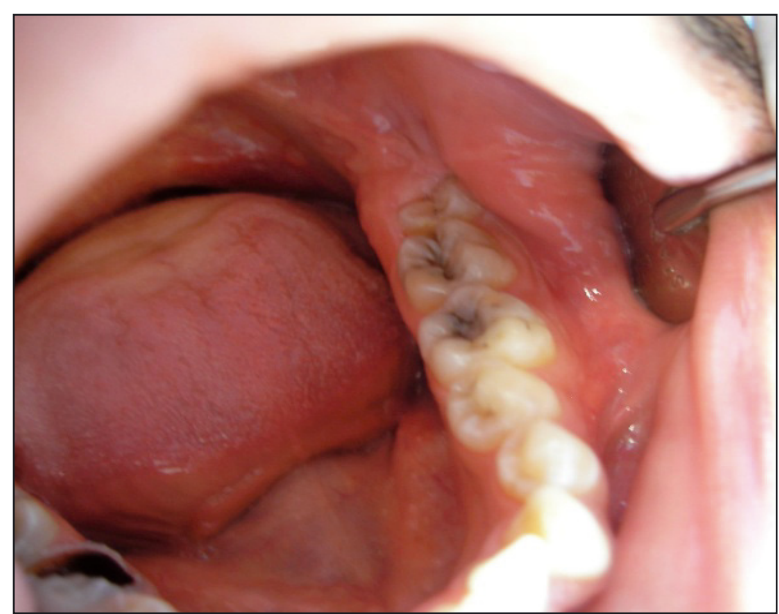

Fig. 2. Vue endobuccale : caries sur 36 et 37 , et 38 enclavée. Fig. 2. Intraoral view: decay of 36 and 37 , and 38 impacted.

une image radio-opaque autour de l'apex de la racine mésiale de cette dent. Après mise en place d'un cône de repérage dans le trajet fistuleux, la radiographie rétro-alvéolaire montre que le cône se dirige vers la zone de furcation.

$\mathrm{Au}$ terme de l'examen clinique et radiologique, le diagnostic de desmodontite chronique sur la 36 est retenu. On procède au traitement habituel : traitement endodontique complet et lavage de la fistule à l'hypochlorite de sodium (Dakin ${ }^{\circledR}$ ) à partir de l'orifice cutané. La radiographie rétro-alvéolaire réalisée après l'obturation canalaire confirme que celle-ci est incomplète sur le tiers apical de la racine mésiale en raison de la calcification préexistante (Fig. 3).

Lors de l'examen de contrôle effectué quinze jours après le traitement endodontique, on n'observe aucune amélioration : l'aspect de la fistule est inchangé. On décide alors de réaliser l'amputation de la racine mésiale de la 36. Après 15 jours, l'évolution semble favorable, mais le patient revient consulter

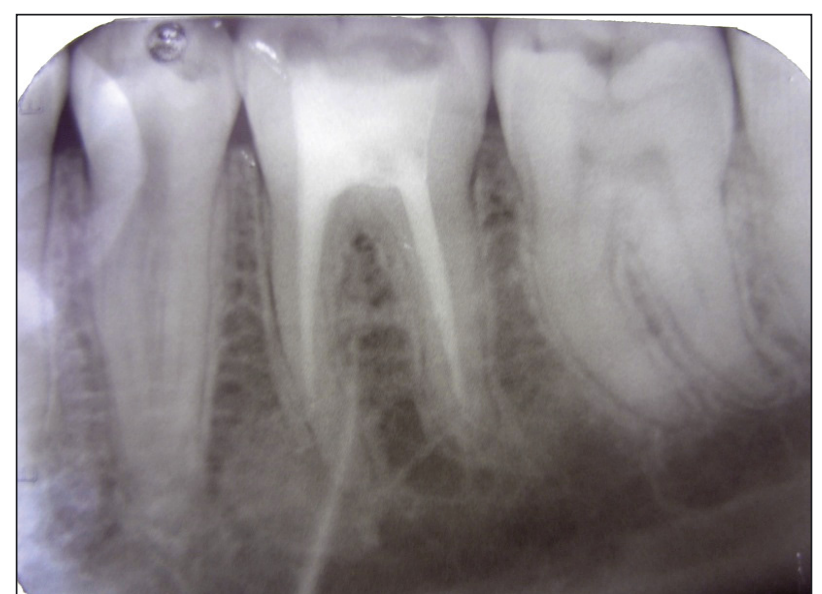

Fig. 3. Obturation canalaire insuffisante de la racine mésiale de la 36. Cône de repérage dirigé vers la zone de furcation.

Fig. 3. Incomplete canal treatment of the mesial root of the 36, tracting cone moving to the furcation.

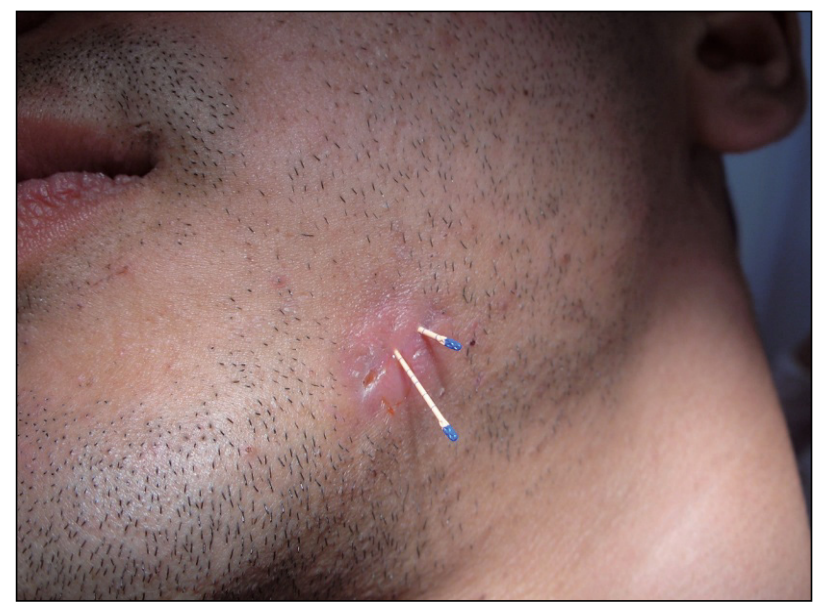

Fig. 4. Après 5 semaines, présence de deux orifices fistulaires. Fig. 4. After 5 weeks, presence of 2 sinus tracts.

une semaine plus tard car une deuxième fistule cutanée est apparue à côté de la précédente. Il n'y a pas de manifestations endobuccales (Fig. 4).

Comme les cônes de repérage semblent se diriger vers la racine distale (Fig. 5), cette racine a été extraite et un trait de fracture longitudinal mis en évidence par la coloration au bleu de méthylène (Fig. 6). Un débridement du trajet fistuleux par voie endobuccale et l'exérèse de l'orifice fistuleux sont réalisés dans la même séance (Figs. 7 et 8). Un mois plus tard, le patient constate la réapparition d'une nouvelle fistule associée à un écoulement purulent (Fig. 9). La persistance de cette fistule conduit à rechercher une autre étiologie : on suspecte une péricoronarite chronique sur la 38 bien qu'il n'y ait pas de signes cliniques évidents (absence de douleurs et d'inflammation). On propose au patient de procéder à l'extraction de la 38. Lors de la réalisation de l'anesthésie péri-apicale, le produit anesthésique s'écoule par la fistule 


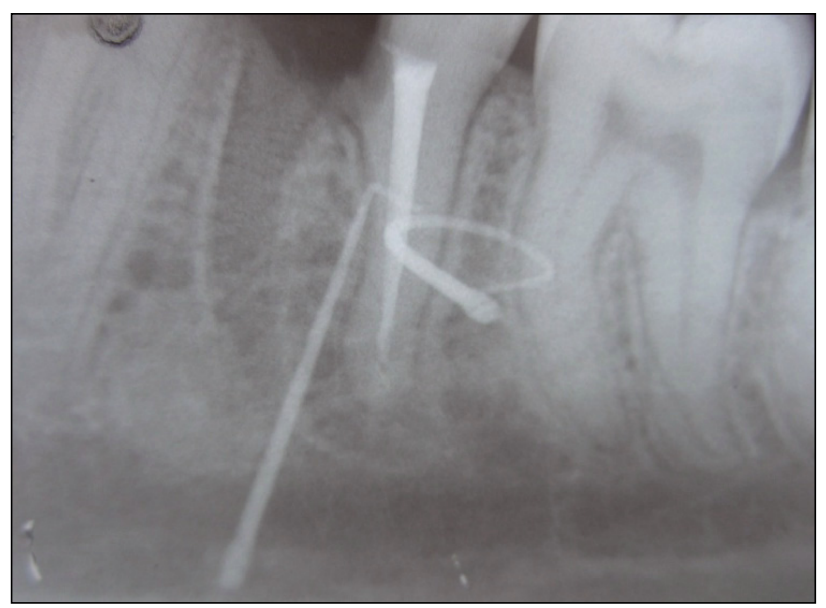

Fig. 5. L'un des cônes de repérage est dirigé vers la racine distale de la 36, l'autre semble entourer la racine distale de la 36.

Fig. 5. One of the tracting cone is directed to the distal root of the 36 , the other seems to circle the distal root of 36 .

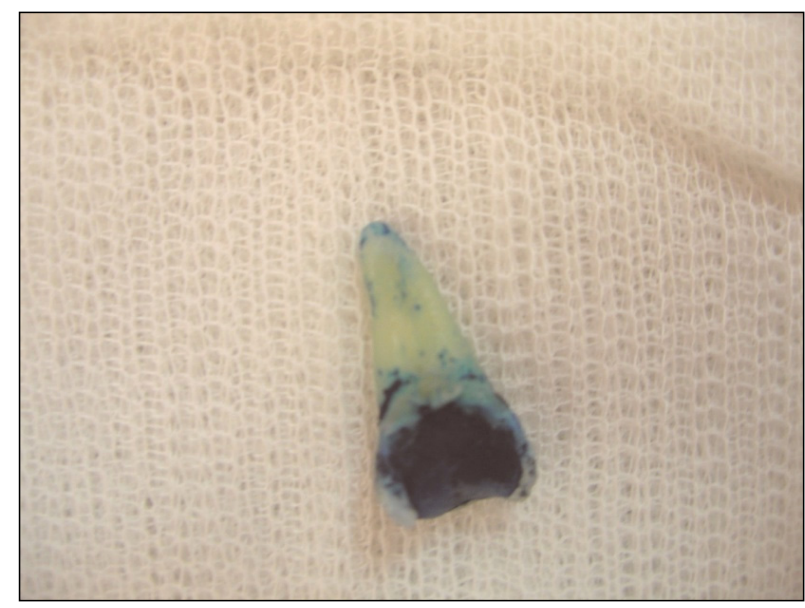

Fig. 6. Mise en évidence du trait de fracture longitudinale sur la racine distale de la 36 par une coloration au bleu de méthylène. Fig. 6. Highlight of the longitudinal fracture line on the distal root of 36 by methylen blue coloration.

(Fig. 10). L'évolution de la fistule cutanée est favorable en une quinzaine de jours. Après un an, il persiste une cicatrice avec une plage d'alopécie (Fig. 11) qui a été éliminée par une intervention de chirurgie plastique.

\section{Discussion}

La conduite à tenir face à une fistule cutanée d'origine dentaire est bien codifiée et l'évolution rapidement favorable après traitement ou extraction de la dent causale. Il est donc primordial de suspecter le diagnostic étiologique dans les meilleurs délais afin de ne pas retarder la prise en charge thérapeutique. L'aspect de la fistule cutanée n'est pas toujours

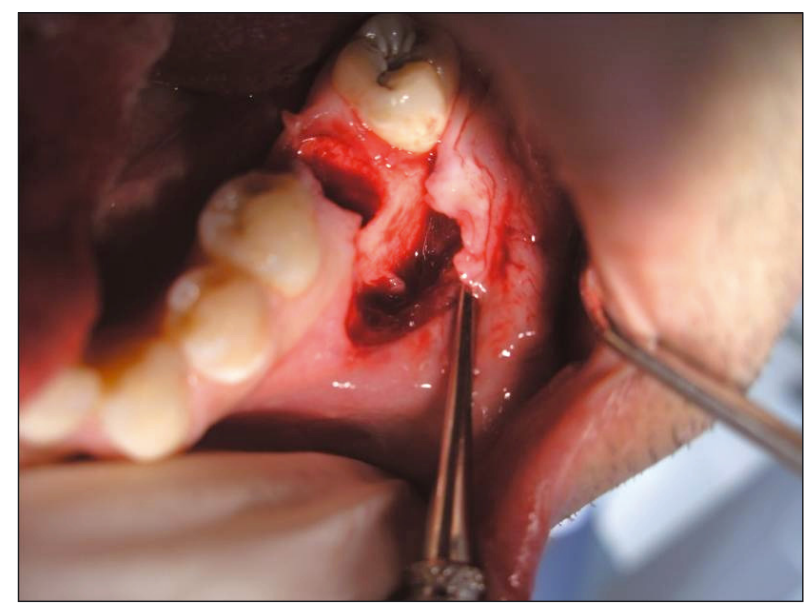

Fig. 7. Débridement mettant en évidence le trajet fistuleux qui part de la zone de furcation.

Fig. 7. Debridement showing sinus tract leading from the furcation area.

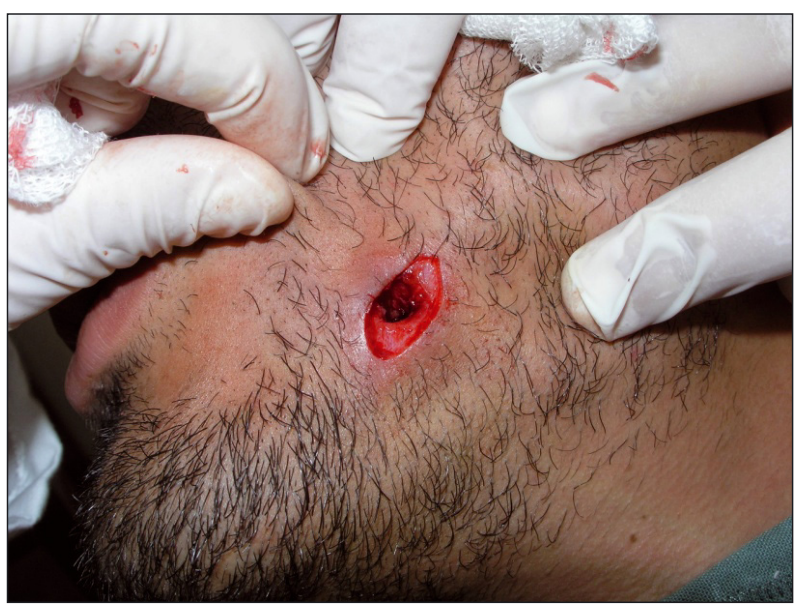

Fig. 8. Exérèse du trajet fistuleux et de la fistule cutanée. Fig. 8. Excision of path and cutaneous sinus tract.

caractéristique, elle peut simuler un granulome pyogénique, une infection cutanée bactérienne ou fongique, une réaction à corps étranger, un carcinome baso-cellulaire ou épidermoïde, ou être assimilée à une fistule secondaire à une ostéomyélite, à une fistule congénitale $[6,7]$. Dans la littérature, on retrouve un cas de fistule cutanée où le diagnostic correct a été effectué seulement 32 ans après l'apparition de la lésion [8]. Parfois, cette fistule est considérée comme une lésion dermatologique et traitée par exérèse chirurgicale, voire jadis par radiothérapie; quelquefois une biopsie apparait comme nécessaire $[9,10]$. Dans le cas rapporté, l'origine dentaire a bien été suspectée mais pas l'existence probable de deux foyers infectieux. A la première consultation, les examens clinique et radiologique orientent vers le foyer endodontique sur la 36. La fistule siège dans la région génienne basse, en regard de la 36 mortifiée et le cône de repérage se dirige vers 


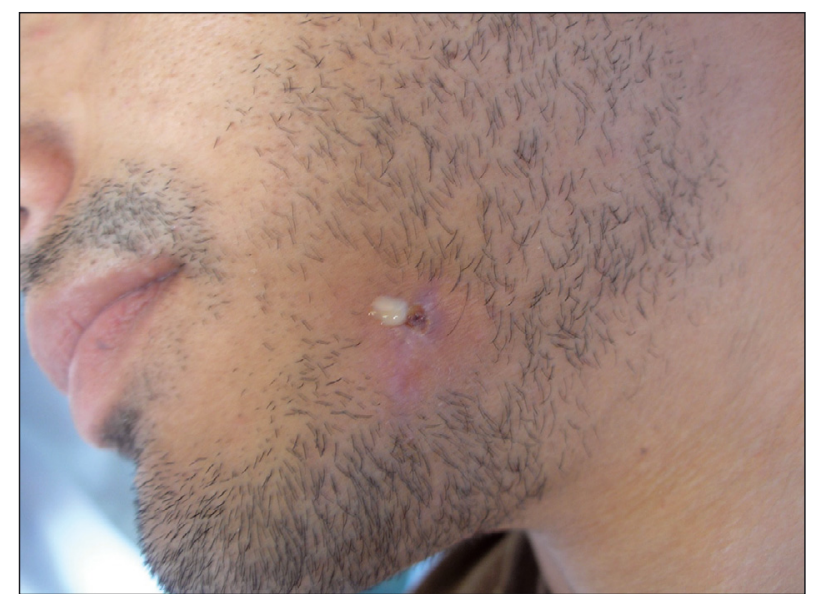

Fig. 9. Après 11 semaines, réapparition de la fistule, associée à un écoulement purulent.

Fig. 9. After 11 weeks reapparition of the fistula, associated to purulent flow.

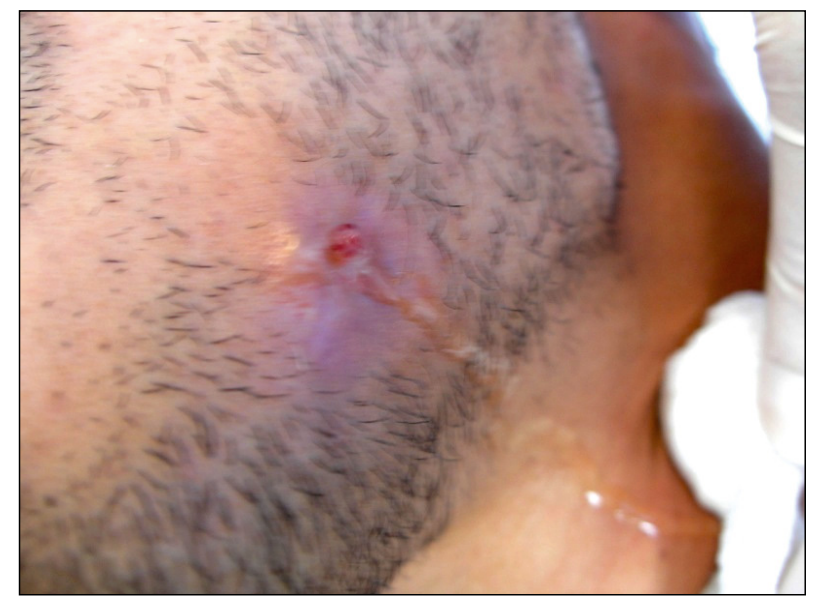

Fig. 10. Ecoulement du liquide anesthésique par la fistule lors l'anesthésie locale pour l'extraction de la 38.

Fig. 10. Anesthesic liquid flow by fistula during local anesthesia for extraction of 38.

la région de la furcation. Selon Slutzky-Goldberg et al. [11] et Brown et al. [12], dans $71 \%$ des cas, la fistule cutanée est secondaire à un foyer infectieux d'origine endodontique. L'amputation de la racine mésiale de la 36 qui présente une obturation insuffisante, a été décidée en l'absence d'évolution favorable dans le délai habituel, normalement entre 5 et 15 jours après l'élimination du foyer infectieux périapical [13]. L'apparition d'une deuxième fistule qui incrimine toujours la 36 a conduit à l'avulsion de sa racine distale. Selon Slutzky-Golberg et al. [11], la présence de plusieurs orifices fistuleux peut être révélatrice d'une fracture radiculaire longitudinale. Dans ce cas, celle-ci a été recherchée par l'examen radiologique et confirmé par la coloration au bleu de méthylène.

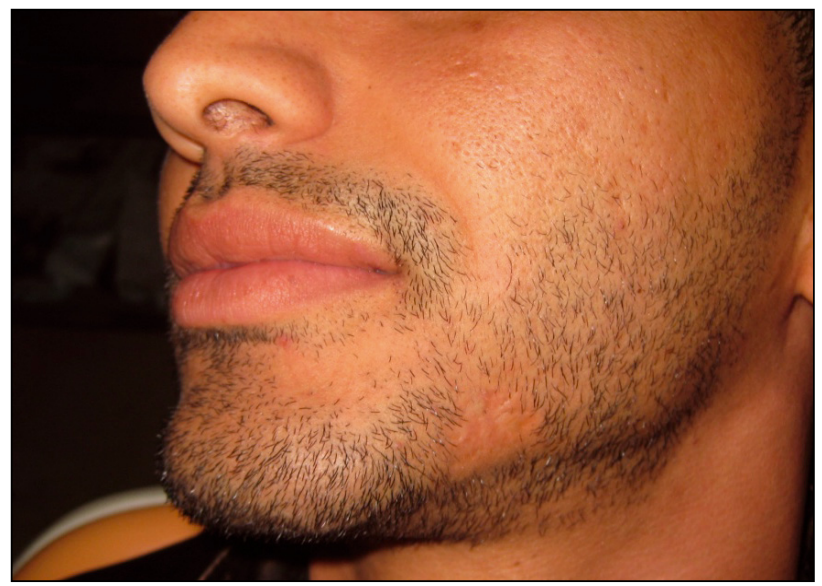

Fig. 11. Aspect de la cicatrice après 1 an d'évolution : plage d'alopécie.

Fig. 11. Appearance of scare after 1year evolution: alopecia area.

La persistance de la fistule cutanée malgré les démarches thérapeutiques entreprises a amené à rechercher une seconde (ou une autre) étiologie. La dent de sagesse, qui constitue le deuxième foyer infectieux susceptible de donner naissance à une fistule génienne basse, n'a pas était mise en cause initialement en l'absence de signes cliniques et radiologiques traduisant une inflammation ou une infection. L'anamnèse, considérée comme primordiale pour la recherche de l'étiologie d'une fistule cutanée, n'a pas permis de suspecter la dent de sagesse [14].

Une péricoronarite peut être à l'origine d'une fistule génienne basse à hauteur de la région prémolaire quand le pus se draine dans la gouttière formée par l'insertion du masséter sur la mandibule. Arrivé dans une zone de moindre résistance dont la limite antérieure correspond au bord postérieur du muscle abaisseur de la commissure, la limite supérieure au bord inférieur du buccinateur, la limite postérieure au bord antérieur du masséter et la limite inférieure au bord basilaire de la mandibule, l'écoulement purulent s'oriente vers la peau et donne naissance à une collection purulente ou à une fistule. En exerçant une pression dans la région péricoronaire de la dent de sagesse, on peut le plus souvent stimuler l'écoulement purulent $[15,16]$.

A posteriori, on peut se demander s'il y avait une ou deux dents causales. L'absence de douleurs à la percussion axiale de la 36, l'absence de cordon dans le fond du vestibule et l'orientation du cône de repérage vers la furcation de la 36 constituent des éléments dont la valeur a probablement été sous-estimée. Le cône qui semble s'enrouler autour de la racine distale (Fig. 5) se trouve probablement dans le trajet fistuleux venant de la 38, et il n'a pas pu franchir l'angle existant entre sa partie antérieure à orientation transversale et sa partie postérieure à orientation sagittale. Au total, il y avait probablement deux foyers infectieux survenant presque simultanément. Ou alors la 38 était l'unique dent causale et c'est seulement la réalisation d'une anesthésie sur la 38 , lors de la 
consultation initiale, qui aurait permis de confirmer d'emblée l'étiologie.

\section{Conclusion}

Le diagnostic clinique d'une fistule cutanée d'origine dentaire est relativement aisé mais il n'en est pas de même pour le diagnostic étiologique. En effet, l'existence de foyers infectieux concomitants peut engendrer des difficultés diagnostiques, même pour un clinicien averti. Si l'indication thérapeutique reste le plus souvent simple - traitement endodontique ou avulsion de la dent causale -, il est parfois nécessaire, pour les fistules anciennes, de procéder à l'exérèse du trajet fistuleux.

\section{Conflits d'intérêt : aucun}

\section{Références}

1. Tidwell E, Jenkins JD, Ellis CD, Hutson B, Cederberg RA. Cutaneous odontogenic sinus tract to the chin. Int Endod $\mathrm{J}$ 1997;30:352-5.

2. Ailianos J. Fistule de la région mentonnière d'origine dentaire. Rev Fr Odonto-Stomatol 1955;2:5.

3. Johnson BR, Remeikis NA, Van Cura JE. Diagnosis and treatment of cutaneous facial sinus tracts of dental origin. J Am Dent Assoc 1999;130:832-6.
4. Sheehan DJ, Potter BJ, Davis LS. Cutaneous draining sinus tract of odontogenic origin: unusual presentation of a challenging diagnosis. Southern Med J 2005;98:250-2.

5. Wilson SW, Ward DJ, Burns A. Dental infections masquerading as skin lesions. Br J Plast Surg 2001;54:358-60.

6. Roldi A, Novaes Pinheiro T, Consolaro A. Nasal sinus tract caused by dental trauma. Brazilian J Dent Traumatolo 2009;1:20-2.

7. Kalaskar RR, Damle SG. Loss of permanent mandibular lateral incisor and canine tooth buds through extaoral sinus: report of case. J Indian Soc Pedod Prev Dent 2006;24:50-2.

8. Bernick SM, Jensen JR. Chronic draining extraoral fistula of 32 years' duration. Oral Surg 1969;27:790-4.

9. Tülin Güleç A, Seçkin D, Bulut S, Safakođlu E. Cutaneous tract of dental origin. Int J Dermatol 2001;40:650-2.

10. Kannan SK, Sandhya G, Selvarani R. Periostitis ossificans. Garre's osteomyelitis radiographic study of two cases. Int J Paediatr Dent 2006;16:59-64.

11. Slutzky-Golberg I, Tsesis I, Slutzky H, Heling I. Odontogenic sinus tracts: a cohort study. Quintessence Int 2009;40:13-8.

12. Brown RS, Jones R, Feimster T, Sam FE. Cutaneous sinus tracts (or emerging sinus tracts) of odontogenic origin: a report of 3 cases. Clin Cosmet Investigat Dent 2010;2:63-7.

13. Varol A, Gülses A. An unusual odontogenic cutaneous sinus tract to the cervical region : a case report. OHDMBSC 2009;8:43-5.

14. Spear KL, Sheridan PJ, Perry HO. Sinus tracts to the chin and jaw of dental origin. J Am Acad Dermatol 1983;8:486-92.

15. Peron JM, Mangez JF. Cellulites et fistules d'origine dentaire. Encycl Med Chir stomatologie/odontologie, 22-033-A-10. Editions scientifiques et médicales, Elsevier SAS, Paris, 2002.

16. Guyot L, Catherine JH, Richard O, Olivi P, Chossegros C. Fistules cutanées d'origine dentaire. Ann Dermatol Venereol 2006;133:725-7. 